\title{
Aortic Aneurysm Eroding into the Spine
}

\author{
T. Konrad Rajab, MD ${ }^{1} \quad$ Miriam W. Beyene ${ }^{2} \quad$ Farhang Yazdchi, MD ${ }^{1} \quad$ Matthew T. Menard, MD ${ }^{1}$
}

1 Division of Vascular and Endovascular Surgery, Department of Surgery, Brigham and Women's Hospital and Harvard Medical School, Boston, Massachusetts

2 Brandeis University, Waltham, Massachusetts

Address for correspondence T. Konrad Rajab, MD, Department of Surgery, Brigham and Women's Hospital, 75 Francis Street, Boston, MA 02115 (e-mail: t.rajab@gmail.com).

AORTA 2018;6:68-69.

\begin{abstract}
Keywords

- aortic aneurysm

- rupture

- contained rupture

- surgery

Aortic aneurysms are usually asymptomatic until catastrophic rupture occurs. Ruptured abdominal aortic aneurysms classically present with acute back pain, shock, and a pulsatile abdominal mass. The natural history of some aortic aneurysms also includes a stage of contained rupture. This occurs when extravasation of blood from the ruptured aneurysm is contained by surrounding tissues. Here, the authors report the case of a chronic contained abdominal aortic aneurysm rupture that resulted in erosion of the spine.
\end{abstract}

Abdominal aortic aneurysms are usually asymptomatic until catastrophic rupture occurs. ${ }^{1}$ Ruptured abdominal aortic aneurysms classically present with acute back pain, shock, and a pulsatile abdominal mass. ${ }^{2}$ The natural history of some aortic aneurysms also includes a stage of contained rupture. This occurs when extravasation of blood from the ruptured aneurysm is contained by surrounding tissues. Here, we report the case of a chronic contained abdominal aortic aneurysm rupture that resulted in erosion of the spine.

A 76-year-old male smoker presented with progressive back pain and a pulsatile abdominal mass. He had a history of coronary artery disease, hypertension, hyperlipidemia, and chronic obstructive pulmonary disease. Physical examination revealed an expansile abdominal mass and diminished femoral pulses. Laboratory testing was notable for a normal white blood cell count, erythrocyte sedimentation rate, and creatinine. Computed tomography demonstrated a $14 \mathrm{~cm}$ juxtarenal abdominal aortic aneurysm (-Fig. 1) eroding into the third and fourth lumbar vertebrae (-Figs. 1 and 2 ). There was also severe iliofemoral occlusive disease. The patient was taken to the operating room for urgent open aortic aneurysm repair. Incision of the aneurysm sac revealed eroded vertebral bodies that were exposed within the aortic lumen (-Fig. 3). There was moderate atherosclerosis but no obvious inflammation. Microbiology specimen did not grow any organisms. The aneurysm was repaired with an aortobifemoral reconstruction using a bifurcated Dacron graft. The postoperative course was unremarkable and the patient was discharged to rehabilitation on postoperative day 5. Orthopaedic consultation recommended conservative management of the spinal erosion without a need for operative stabilization. He was at his baseline 12 months following his repair.

Ruptured aortic aneurysm has a high mortality rate. In a large prospective series of patients with ruptured aneurysms, $25 \%$ of patients died before reaching a hospital and $51 \%$ died at the hospital before undergoing surgery. ${ }^{3}$ The consequences of abdominal aortic aneurysm rupture depend on the anatomic location of the rupture. Free rupture into the

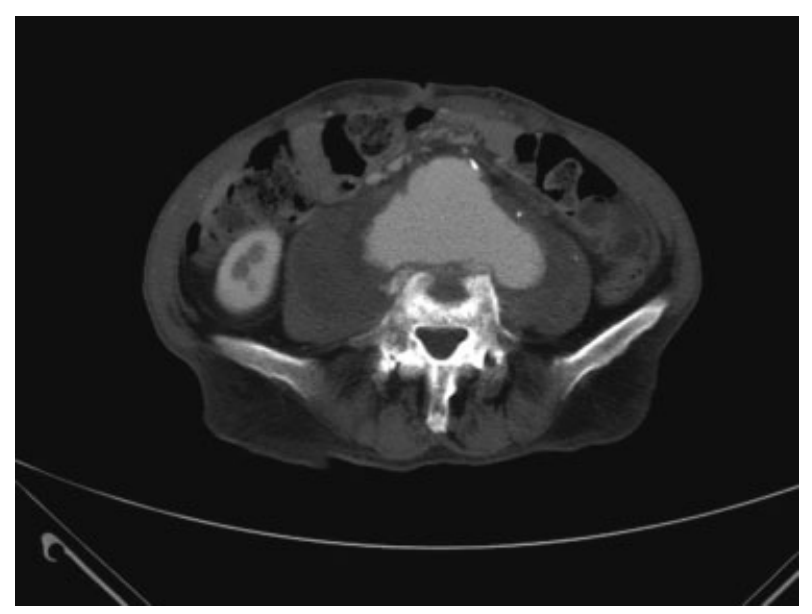

Fig. 1 Computed axial tomography shows the $14 \mathrm{~cm}$ abdominal aortic aneurysm with mural thrombus. received

May 12, 2017

accepted after revision

June 12, 2018
DOI https://doi.org/

10.1055/s-0038-1669416. ISSN 2325-4637.
Copyright $\odot 2018$ by Thieme Medical Publishers, Inc., 333 Seventh Avenue, New York, NY 10001, USA.

Tel: +1(212) 584-4662.
License terms

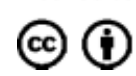




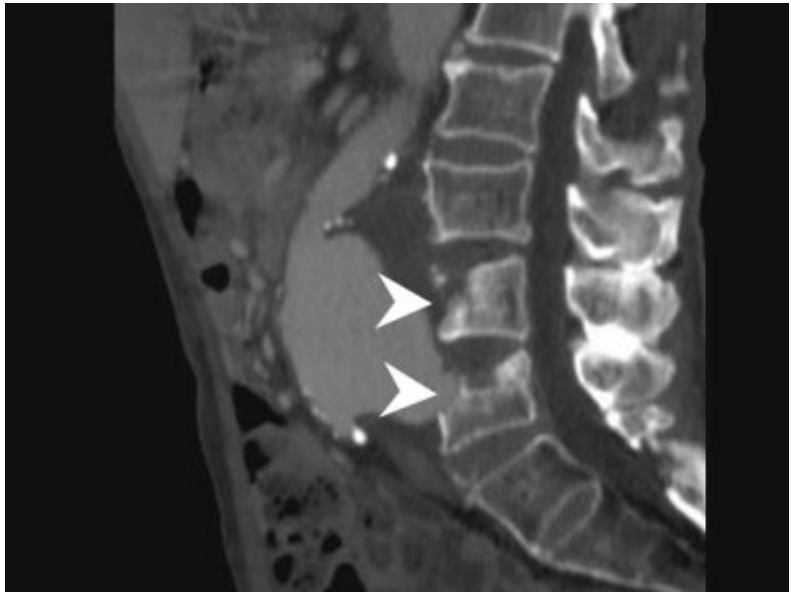

Fig. 2 Bone window images of the sagittal reconstruction show the aneurysm eroding the spine (arrowheads).

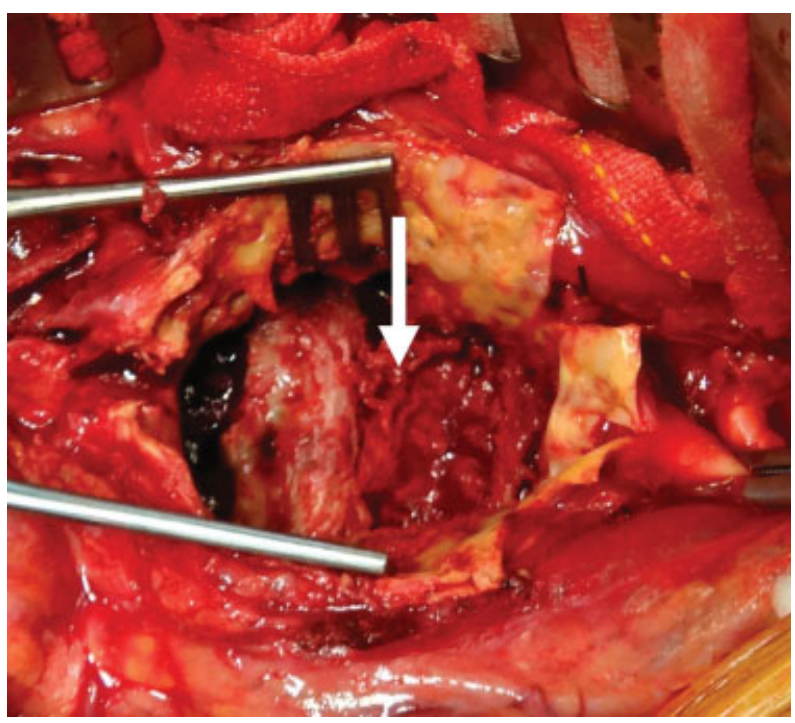

Fig. 3 Intraoperative photograph shows the incised aneurysm sac (arrow), and eroded vertebral bodies that are exposed inside the aortic aneurysm. peritoneal cavity is rapidly fatal. Contained rupture occurs when extravasation of blood is contained by surrounding tissues. Depending on the quality of the surrounding tissue, chronic contained rupture may be present over a prolonged period of time. The duration of this stage depends on the strength of the tissue containing the hematoma. Posterior extension of the rupture can erode the spine, as occurred in this patient and others described in the literature. ${ }^{4,5}$ Alternatively, local extension of vertebral osteomyelitis or discitis can cause mycotic aortic aneurysm formation. These pathologies result in atypical presentations of abdominal aortic aneurysms.

\section{Conflict of Interest}

The authors declare no conflict of interest related to this article.

\section{Funding}

None

\section{Acknowledgment}

None

\section{References}

1 Sakalihasan N, Limet R, Defawe OD. Abdominal aortic aneurysm. Lancet 2005;365(9470):1577-1589

2 Isselbacher EM. Thoracic and abdominal aortic aneurysms. Circulation 2005;111(06):816-828

3 Brown PM, Pattenden R, Vernooy C, Zelt DT, Gutelius JR. Selective management of abdominal aortic aneurysms in a prospective measurement program. J Vasc Surg 1996;23(02):213-220, discussion 221-222

4 Oldenburg WA, Almerey T. Erosion of lumbar vertebral bodies from a chronic contained rupture of an abdominal aortic pseudoaneurysm. J Vasc Surg 2016;2(04):197-199

5 Li Y, Li L, Zhang D, Wang X, Sun W, Wang H. A contained ruptured abdominal aortic aneurysm presenting with vertebral erosion. Ann Vasc Surg 2017;41(41):279.e13-279.e17 